\title{
Pump-induced refractive index modulation and dispersions in Er3+-doped fibers
}

\author{
Thirstrup, Carsten; Shi, Yuan
}

Published in:

Journal of Lightwave Technology

Link to article, DOI:

$10.1109 / 50.495152$

Publication date:

1996

Document Version

Publisher's PDF, also known as Version of record

Link back to DTU Orbit

Citation $(A P A)$ :

Thirstrup, C., \& Shi, Y. (1996). Pump-induced refractive index modulation and dispersions in Er3+-doped fibers. Journal of Lightwave Technology, 14(5), 732-738. https://doi.org/10.1109/50.495152

\section{General rights}

Copyright and moral rights for the publications made accessible in the public portal are retained by the authors and/or other copyright owners and it is a condition of accessing publications that users recognise and abide by the legal requirements associated with these rights.

- Users may download and print one copy of any publication from the public portal for the purpose of private study or research.

- You may not further distribute the material or use it for any profit-making activity or commercial gain

- You may freely distribute the URL identifying the publication in the public portal

If you believe that this document breaches copyright please contact us providing details, and we will remove access to the work immediately and investigate your claim. 


\title{
Pump-Induced Refractive Index Modulation and Dispersions in $\mathrm{Er}^{3+}$-Doped Fibers
}

\author{
Carsten Thirstrup, Yuan Shi, and Bera Pálsdóttir
}

\begin{abstract}
A novel measurement system provides determination of pump induced phase shifts in erbium doped fibers with an accuracy of $\sim \pi / 20$. Using this system, a systematical analysis of the pump induced modulation of the refractive index and dispersions for a signal at $1550 \mathrm{~nm}$ and a pump at $980 \mathrm{~nm}$ is reported. The analysis contains measurements of pump induced refractive index changes as function of wavelength, pump power, and doping concentration. A model taking account of the contribution to the refractive index changes from optical transitions between ${ }^{4} \mathbf{I}_{15 / 2}$ states and ${ }^{4} \mathbf{I}_{13 / 2}$ states in $\mathrm{Er}^{3+}$ yields good agreement to experimental results apart from a wavelength independent offset. The offset is interpreted to originate from high energetic optical transitions. The results show that for a large refractive index modulation, a short and highly doped fiber should be used with limited amplified spontaneous emission effect. In optical communication systems comprising erbium doped fiber amplifiers, a tradeoff between dispersion and amplification must be made.
\end{abstract}

\section{INTRODUCTION}

$\mathbf{T}$ HE RESONANT enhanced optical nonlinearities and dispersions caused by erbium ions $\left(\mathrm{Er}^{3+}\right)$ in a glass matrix are currently attracting considerable interest [1-5] since they influence the performance of erbium doped fiber amplifiers (EDFA's). which have important applications in soliton transmission systems, short pulse fiber lasers, and cascaded EDFA's in transmission systems. In order to optimize these components and systems, it is therefore important to understand how the nonlinearities and dispersions depend on fiber parameters such as doping concentration, pump power, and fiber dimensions.

Pumping an $\mathrm{Er}^{3+}$-doped $\mathrm{SiO}_{2}$ fiber at $980 \mathrm{~nm}$, Fleming et al. [1] have measured phase shifts of light for wavelengths close to the resonant wavelength of $1530 \mathrm{~nm}$, where the amplification is maximum. The phase shifts were measured using a fiber Mach-Zehnder interferometer; however, the authors did not succeed in determining the phase shifts accurately with the method because of many factors, such as power levels in each arm and fringe visibility due to polarization mismatch. Spectral dependence of output coupling ratio also had to be taken into account [1]. Other authors have determined pump induced phase shifts indirectly from the switching characteristics of a twin core fiber coupler [2-5]. Using this technique, the phase

Manuscript received July 14, 1994; revised January 25, 1996.

C. Thirstrup is with the Mikroelektronik Centret, Technical University of Denmark, Building 345 East 2800 Lyngby, Denmark. He is also with Surface and Interface Laboratory, RUKEN, Saitama, Japan.

Y. Shi is with the Mikroelektronik Centret, Technical University of Den mark. 2800 Lyngby, Denmark. He is also with E-TEK Dynamics, Inc., San Jose, CA 95131 USA.

B. Pálsdóttir is with the Lycom A/S, NKT All 75, 2605 Brøndby, Denmark. Publisher Item Identifier S 0733-8724(96)03545-1. shifts have been measured for a) a pump wavelength of 1480 $\mathrm{nm}$ and a signal wavelength in the wavelength region $900-960$ $\mathrm{nm}$ [2], [5] and b) for a pump wavelength of $514.5 \mathrm{~nm} / 980$ $\mathrm{nm}$ and a signal wavelength of $5145 \mathrm{~nm}$ [3], [4].

In this paper, an improved direct measurement technique for determining the pump induced phase shifts in $\mathrm{Er}^{3}$. doped fibers is reported. The $\mathrm{Er}^{3+}$ ions are pumped at a wavelength of $980 \mathrm{~nm}$ and the phase changes are measured at wavelengths in the vicinity of the resonant transition at $1530 \mathrm{~nm}$ (from ${ }^{4} \mathrm{I}_{15 / 2}$ states to $\mathrm{I}_{13 / 2}$ states). The resonant transition enhances the refractive index modulation, and the corresponding optical nonlinearity is significantly larger than Kerr type nonlinearities, but at the expense of speed. The speed of the resonant nonlinear effect in $\mathrm{Er}^{3}$ - doped fibers is limited by the fluorescence lifetime of the metastable energy level. However, it has been observed that $\mathrm{Er}^{3+}$-doped fibers also comprise a considerable nonresonant term to the optical nonlinearity.

The measurement system consists of a Mach-Zehnder interferometer using a polarization maintaining $3 \mathrm{~dB}$ fiber coupler and a signal wavelength in the range $1500-1600 \mathrm{~nm}$. One arm of the interferometer includes an $\mathrm{Er}^{3+}$-doped fiber pumped at $980 \mathrm{~nm}$, which causes a phase change at the signal wavelength. A differential detection scheme improves the sensitivity of the phase measurements and eliminates influence of amplified spontaneous emission (ASE). Using a linear modulation in time of the reference arm by means of a piezoelectric crystal yields a measurement accuracy of $\sim \pi / 20$. The measurement technique is relatively insensitive to variations in external parameters such as polarization states and power levels in the two arms of the Mach-Zehnder interferometer, and it could also be useful in investigating other doped fibers, such as semiconductor doped glasses and phosphorous-oxygen hole centers [6].

Employing the measurement system, a systematical investigation of the dependence of refractive index changes in $\mathrm{Er}^{3+}$-doped fibers upon wavelength, pump power, and doping concentration is carried out in this paper. The results can be interpreted using a model that takes account of the contribution to the refractive index changes from optical transitions between ${ }^{4} \mathrm{I}_{15 / 2}$ states and ${ }^{4} \mathrm{I}_{13 / 2}$ states. The model is based on measured spectra of absorption and emission cross sections, and it yields good agreement to the experimental results apart from a wavelength independent offset. The refractive index modulations are compared with the behavior of the amplified spontaneous emission, and the dispersions are derived from the measurements of the pump induced refractive index changes. 


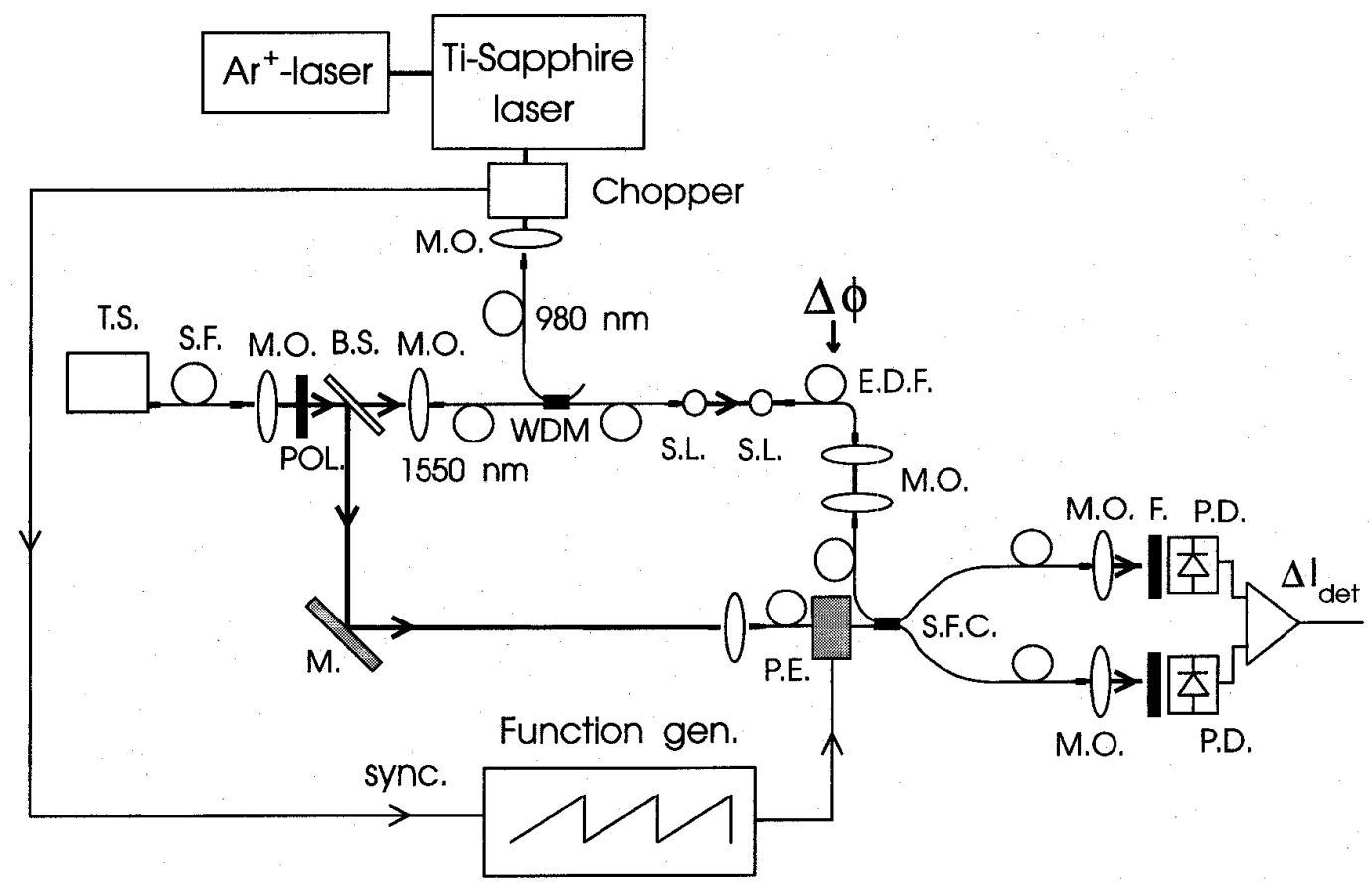

Fig. 1. Schematic of the setup for directly measuring pump induced phase modulation in $E \mathbf{r}^{3+}$-doped fibers. T.S.: tunable semiconductor laser; S.F.: single mode fiber; M.O.: microscope objective; POL.: polarizer; B.S.: beam splitter; M.: mirror; WDM: $980 \mathrm{~nm} / 1550 \mathrm{~nm}$ wavelength division multiplexer;

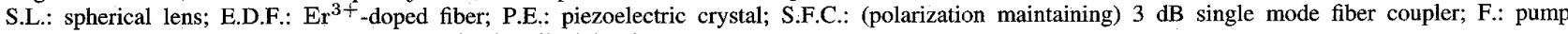
block filter; P.D.: photodiode. The system is described in the text.

\section{EXPERIMENTAL SETUP}

The system employed for measuring pump induced phase shifts in $\mathrm{Er}^{3+}$-doped fibers is shown in Fig. 1. Pump light at $980 \mathrm{~nm}$ from a Ti:sapphire laser pumped by an $\mathrm{Ar}^{+}$-laser is chopped and coupled into the $\mathrm{Er}^{3+}$-doped fiber (E.D.F.) via a wavelength division multiplexer (WDM). The $\mathrm{Er}^{3+}$-doped fiber is mounted in one arm of a Mach-Zehnder interferometer. Circular polarized signal light from a tunable GaInAsP laser with wavelengths from 1500 to $1600 \mathrm{~nm}$ and output intensity of $\sim 30 \mu \mathrm{W}$ is coupled into the Mach-Zehnder interferometer with $\sim 80 \%$ of the power coupled into the $\mathrm{Er}^{3+}$-doped fiber via the WDM and $\sim 20 \%$ coupled into the reference arm. Light from both arms of the interferometer is collimated and coupled into corresponding single mode polarization maintaining fiber arms of a $3 \mathrm{~dB}$ coupler, and two InGaAs photodiode detectors are used to monitor the light from the two output ports of the fiber coupler. Using a differential detection scheme eliminates influence of ASE and increases the detection sensitivity. The phase in the reference arm is linearly modulated in time, $\phi_{m}=\omega t$, by means of a piezoelectric crystal. This results in a response of the detected signal, which in the case of no pump can be written

$$
\Delta I_{\text {det }} \propto \cos \left(\omega t+\phi_{0}\right)
$$

and in presence of the pump

$$
\Delta I_{\text {det }} \propto \cos \left(\omega t+\phi_{0}+\phi_{p}\right)
$$

where $\phi_{0}$ is an arbitrary phase and $\phi_{p}$ is the pump induced phase shift of the signal. $\phi_{p}$ is determined by measuring the phase difference of the cosine waveforms before and after the pump is turned on by the optical chopper. Since the technique is based on measuring phase differences rather than amplitudes, it is relatively insensitive to changes in power levels and polarization states of the two interferometric arms and spectral dependence of the output coupling ra:io. The accuracy of the phase measurement is limited by the resolution of the digital oscilloscope which displays the waveforms and it is estimated to be $\pi / 20$. This is a large improvement in accuracy compared to previous reports of direct meast rements of the pump induced phase shifts [1]. The linearity of the piezoelectric modulation has been controlled by measuring the phase changes for different periods of the cosine waveform. The changes were found to be within the measuring accuracy for each period.

\section{MODEL}

\section{A. Analysis Based on Absorption and Emission Cross Sections}

From measured absorption and emission cross sections, the resonant contribution from the optical transitions between the ground states $\left({ }^{4} \mathrm{I}_{15 / 2}\right)$ and the metastable states $\left({ }^{4} \mathrm{I}_{13 / 2}\right)$ in $\mathrm{Er}^{3+}$ to the change in the refractive index can be modeled as follows. Using the Ladenburg-Fuchtbauer relations [7], the room temperature absorption and fluorescence spectral profiles can been converted into the absorption cross section $\sigma_{c}(\lambda)$ and emission cross section $\sigma_{e}(\lambda)$ as function of wavelength $\lambda$. Fig. 2 shows the results at room temperature for a silica fiber with an $\mathrm{Er}^{3+}$-concentration of $0.7 \times 10^{19} \mathrm{~cm}^{-3}$ and $\mathrm{Al}$ - and $\mathrm{La}^{3+}$-codoping where the fluorescence life time was assumed to be $10 \mathrm{~ms}$.

In the unsaturated regime, the imaginary part of the complex susceptibility of $\mathrm{Er}^{3+}$ originating from optical trınsitions 


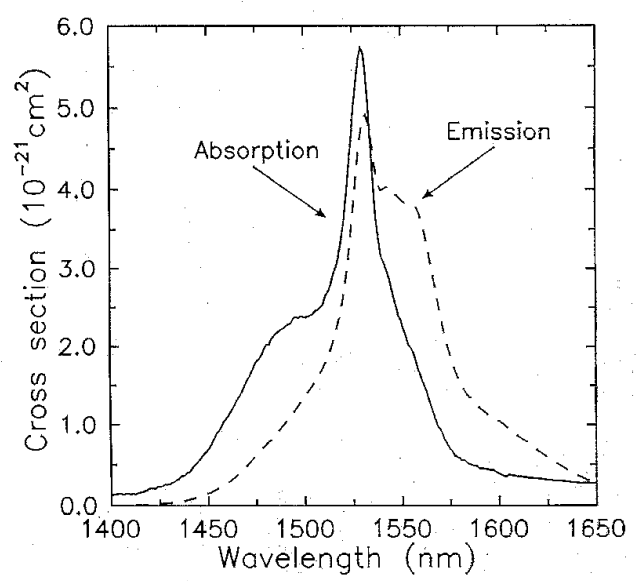

Fig. 2. Room temperature absorption and emission cross-section spectra measured for an $\mathrm{Er}^{3+}$-doped fiber with an $\mathrm{Er}^{3+}$-concentration of $0.7 \times 10^{19} \mathrm{~cm}^{-3}$.

between ${ }^{4} \mathrm{I}_{15 / 2}$ states and ${ }^{4} \mathrm{I}_{13 / 2}$ states has been derived by Desurvire [8];

$$
\chi^{\prime \prime}(\lambda, z)=-n \frac{\lambda}{2 \pi}\left[N_{2}(z) \sigma_{e}(\lambda)-N_{1}(z) \sigma_{a}(\lambda)\right]
$$

where $n$ is the refractive index of the material, $N_{1}(z)$ and $N_{2}(z)$ are the average populations of the ${ }^{4} \mathrm{I}_{15 / 2}$ energy level and the ${ }^{4} \mathrm{I}_{13 / 2}$ energy level at the position $z$ [8]

$$
\begin{aligned}
N_{1}(z) & =\frac{N}{1+\frac{P_{\text {pump }}(z)}{P_{t h}}}, \\
N_{2}(z) & =\frac{P_{\text {pump }}(z)}{P_{t h}} \frac{N}{1+\frac{P_{\text {pump }}(z)}{P_{t h}}}
\end{aligned}
$$

where $N$ is the doping concentration, $P_{\text {pump }}(z)$ is the pump power at $z$, and $P_{t h}$ is a threshold pump power.

The pump induced change in the refractive index $\Delta n$ can then be obtained from

$$
\Delta n(\lambda)=\frac{\Gamma_{S}}{2 n L} \int_{0}^{L}\left[\chi_{P}^{\prime}\left(\lambda, z^{\prime}\right)-\chi_{0}^{\prime}\left(\lambda, z^{\prime}\right)\right] d z^{\prime}
$$

where $L$ is the length of the fiber, $\Gamma_{S}$ is the confinement factor of the signal, $\chi_{P}^{\prime}$ and $\chi_{0}^{\prime}$ are the real parts of the complex susceptibility with and without the pump, respectively. For each $z^{\prime}$ along the fiber, $\chi_{P}^{\prime}\left(\chi_{0}^{\prime}\right)$ can readily be obtained by performing the Kramers-Kronig transform [9] on the corresponding imaginary part of the susceptibility $\chi_{P}^{\prime \prime}\left(\chi_{0}^{\prime \prime}\right)$ which is given by (1).

\section{B. Analytical Expression}

An analytical expression for $\chi_{i}^{\prime}(i=P, 0)$ can be obtained by assuming a two level laser system and that the optical transitions have Lorentzian lineshapes [10]

$$
\chi_{i}^{\prime}(\lambda, z)=-\left[N_{1}(z)-N_{2}(z)\right] \frac{\lambda^{3}}{16 \pi^{2} n \tau_{2}} g_{12}\left(\nu-\nu_{0}\right)
$$

where $\nu$ is the optical frequency and $\nu_{0}$ is the resonance frequency, $\tau_{2}$ is the lifetime of the metastable state and $g_{12}\left(\nu-\nu_{0}\right)$ is the lineshape function;

$$
g_{12}\left(\nu-\nu_{0}\right)=\frac{1}{\pi} \frac{\left(\nu-\nu_{0}\right)}{\left(\frac{\Gamma_{12}}{2}\right)^{2}+\left(\nu-\nu_{0}\right)^{2}}
$$

$\Gamma_{12}$ being the full width half maximum of the transition.

It is now assumed that the pump power decays exponentially. along the fiber with an absorption coefficient $\alpha_{p} ; P_{\text {pump }}(z)=$ $P_{\text {pump }} \exp \left(-\alpha_{p} z\right)$. Using the expressions for $N_{1}$ and $N_{2}$ in (2), inserting (4) into (3) and performing the integration, yield

$$
\begin{aligned}
& \Delta n(\lambda)=N \frac{\Gamma_{S} \lambda^{3}}{16 \pi^{2} n^{2} \tau_{2} \alpha_{P} L} g_{12}\left(\nu-\nu_{0}\right) \\
&\left\{\alpha_{P} L-\log \frac{P_{\text {pump }}+P_{t h} e^{\alpha_{P} L}}{P_{\text {punp }}+P_{t h}}\right\}
\end{aligned}
$$

It is observed from the above expression that the sign of $\Delta n$ is determined by the lineshape function $g_{12}\left(\nu-\nu_{0}\right)$.

A cross phase optical nonlinearity from the pump to the signal $n_{s p}$ can be defined from

$$
\Delta n=n_{s p} \frac{P_{\text {pump }}}{A}
$$

where $\Delta n$ is the change in the refractive index for the signal as defined in (6) and $A$ is the effective area of the pump guided in the fiber. In order to estimate an order of magnitude of the optical nonlinearity, $n_{s p}$ can be calculated from (6) and (7) assuming $P_{\text {pump }}=P_{t h}=1 \mathrm{~mW}$ and inserting typical parameters for an erbium doped fiber: $N=$ $10^{19} \mathrm{~cm}^{-3}, \lambda=1.55 \mu \mathrm{m}, \Gamma_{s}=1, \alpha_{P} L=1, n-1.5, \tau_{2}=$ $10 \mathrm{~ms} ; A=4 \times 10^{-7} \mathrm{~cm}^{2}, g(\nu)=10^{-14} \mathrm{~s}$. The result is, $n_{s p}=0.14 \times 10^{-10} \mathrm{~cm}^{2} / \mathrm{W}$, which is about five orders of magnitude larger than the Kerr-effect for pure silica [11]. The time response of the optical nonlinearity is limited by the life time of the metastable energy level which is approximately 10 ms. A large nonresonant nonlinearity has also been observed in the erbium-doped fibers. This will be discussed in the following section.

\section{RESULTS AND DISCUSSIONS}

According to $(6)$, an increase in the pump power $P$ pump causes a corresponding change in the refractive index. Using the expression;

$$
\Delta n\left(P_{\text {pump }}\right)=\frac{\Delta \phi\left(P_{\text {pump }}\right) \lambda}{2 \pi L}
$$

the pump-induced change in the refractive index $\Delta n\left(P_{\text {pump }}\right)$ can be derived from measurements of the pump induced phase change in the fiber $\Delta \phi\left(P_{\text {pump }}\right)$. Three $\mathrm{Er}^{3+}$-doped fibers with Al- and $\mathrm{La}^{3+}{ }^{3+}$-codopants are investigated. For each fiber $A, B$ and $C$, the $\mathrm{Er}^{3+}$-concentration and fiber length are depicted in Table I. Fig. 3 shows (a) measured and (b) calculated change in the refractive index as function of wavelength for fiber $A$ for three different pump powers, $P_{\text {pump }}=1.0 \mathrm{~mW}$ (circle symbols and solid curves), $P_{\text {pump }}=2.2 \mathrm{~mW}$ (square symbols and dashed curves) and $P_{\text {pump }}=4.2 \mathrm{~mW}$ (triangular symbols and dashdotted curves). The curves in Fig. 3(b) are calculated from the model based on the absorption and emission cross 


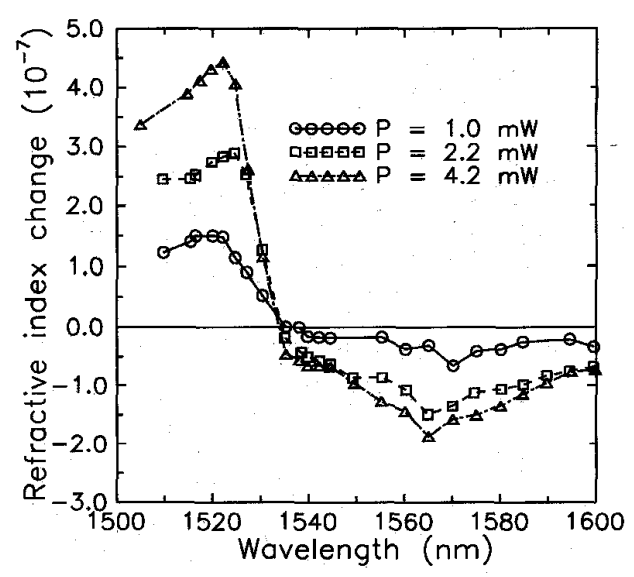

(a)

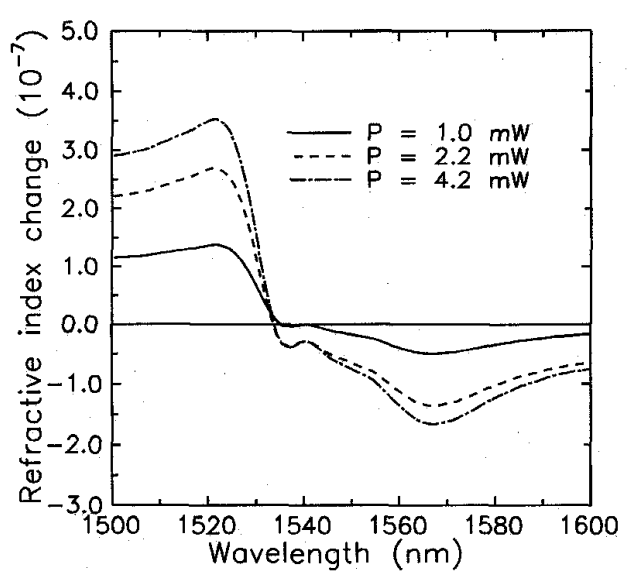

(b)

Fig. 3. Measured (a) and calculated (b) refractive index change as function of signal wavelength for fiber $A\left(\mathrm{Er}^{3+}\right.$-doping concentration of $0.7 \times 10^{19} \mathrm{~cm}^{-3}$ ) at an absorbed pump power of $1.0 \mathrm{~mW}$ (circular symbols and solid curves), $2.2 \mathrm{~mW}$ (square symbols and dashed curves) and $4.2 \mathrm{~mW}$ (triangular symbols and dashdotted curves). The wavelength of the pump power is $980 \mathrm{~nm}$. The threshold pump power $P_{t h}$ has been set equal to 0.8 $\mathrm{mW}$ and the offsets for the calculated solid; dashed, and dashdotted curves in (a) have been set equal to $0.4 \times 10^{-7}, 0.8 \times 10^{-7}$ and $1.1 \times 10^{-7}$, respectively (see text).

sections for transitions between ${ }^{4} \mathrm{I}_{15 / 2}$ states and ${ }^{4} \mathrm{I}_{13 / 2}$ states in $\mathrm{Er}^{3+}$ as described in section IIIA. In order to fit the data, $P_{t h}$ is set equal to $0.8 \mathrm{~mW}$ for all three curves, and a constant of $0.4 \times 10^{-7}, 0.8 \times 10^{-7}$ and $1.1 \times 10^{-7}$, is added to the solid, the dashed, and the dashdotted curve, respectively. The presence of this offset in $\Delta n$ implies that besides the optical transitions between ${ }^{4} \mathrm{I}_{15 / 2}$ states and ${ }^{4} \mathrm{I}_{13 / 2}$ states there are other contributions to the optical nonlinearity. When the offsets are subtracted, the changes in the refractive index are positive below the resonant wavelength $\lambda_{0}=1530 \mathrm{~nm}\left[\nu>\nu_{0}\right.$ in (5)] and negative above the resonant wavelength $\left[\nu<\nu_{0}\right.$ in (5)] as expected from (5) and (6).

A method often used to determine refractive index changes $\Delta n$, is based on employing the Kramers-Kronig transform on experimental absorption data. For the electro-optical effect in III-V semiconductor quantum wells [12], this technique has
TABLE I

Parameters for the Fibers Used IN THE ExPERIMENTS

\begin{tabular}{ccc}
\hline Fibre & $\begin{array}{c}\text { Length } \\
{[\mathrm{cm}]}\end{array}$ & $\begin{array}{c}\mathrm{Er}^{3+} \text {-conc. } \\
{\left[\mathrm{cm}^{-3}\right]}\end{array}$ \\
\hline A & 127 & $0.7 \times 10^{19}$ \\
B & 81 & $2 \times 10^{19}$ \\
C & 35 & $8 \times 10^{19}$ \\
\hline
\end{tabular}

provided good agreement to direct measurements of $\Delta n$. In Fig. 4(a), measurements of the absorption coefficient for fiber $A$ are illustrated for increasing pump powers as indicated. The measurements were carried out using an InGaAsP laser as the signal source which could be tuned from 1500 to $1600 \mathrm{~nm}$. Performing the Kramers-Kronig transform on the change in absorption for a change in pump power from $P_{\text {pump }}=0$ to $2.2 \mathrm{~mW}$ yields $\Delta n$ as shown by square symbols in Fig. 4(b). In order to compare the data with the corresponding results obtained from direct measurements of $\Delta n$, an offset in $\Delta n$ of $0.8 \times 10^{-7}$ (the same as the value used in Fig. 3(b) for a pump power of $2.2 \mathrm{~mW}$ ) has been added to the data in Fig. 4(b). The direct measurement of $\Delta n$ for a pump power of $2.2 \mathrm{~mW}$ is shown as triangular symbols. Considering the narrow range of wavelengths used in the Kramer;-Kronig calculation, there is a fairly good agreement between the two curves for wavelengths longer than $\lambda \sim 1520 \mathrm{~nm}$. However, the considerable absorption change below this wavelength (see Fig. 4(a)) provides a negative contribution to $\Delta n$, and since this contribution is not taken into account in Fig. 4(b) a larger discrepancy is observed at shorter wavelengths.

Fig. 5 shows (a) the measured change in refractive index $\Delta n$ and (b) the calculated $\Delta n$ as function of wavelength for the fibers $A, B$ and $C$ (see Table I) with different $\mathrm{Er}^{3+}$ concentrations, $0.7 \times 10^{19} \mathrm{~cm}^{-3}$ (square symbols and solid curves), $2.0 \times 10^{19} \mathrm{~cm}^{-3}$ (cross symbols and dashecl curves) and $8.0 \times 10^{19} \mathrm{~cm}^{-3}$ (triangular symbols and dashdotted curves). The lengths of the fibers are 127,81 and $35 \mathrm{~cm}$ (see Table I), respectively, and the absorbed pump power is $4.2 \mathrm{~mW}$ in each case. It is observed that $\Delta n$ increases for ircreasing $\mathrm{Er}^{3+}$-concentration, but in contrary to the prediction in (6) the relationship is not linear up to high doping concentrations. As a consequence, in order to fit the experimental data the threshold pump power $P_{t h}$ has been set equal to $0.8 \mathrm{~mW}$ for the weakly doped fibers $A$ and $B$ and equal to $1.5 \mathrm{~mW}$ for fibe: $C$ with the highest $\mathrm{Er}^{3+}$-concentration. The higher $P_{t h}$ for fiber $C$ could be due to ion-ion interaction [13-16]. The offset in $\Delta n$ has been set equal to $1.1 \times 10^{-7}$ for fiber $A, 3.0 \times 10^{-7}$ for fiber $B$, and $4.8 \times 10^{-7}$ for fiber $C$. The offsets are $17.3,19.5$, and $24.1 \%$ of the peak to trough pump induced $\Delta n$ for fiber $A, B$ and $C$, respectively. This suggests that a higher doping concentration yields a relatively higher offset in $\Delta n$.

Fleming et al. [1] have estimated an offset of $1.7 \times 10^{-7}$ in $\Delta n$ near $1550 \mathrm{~nm}$ which is $57 \%$ of the pump induced peak to trough $\Delta n$ of $3 \times 10^{-7}$ for a $150 \mathrm{~cm}$ long erbium doped fiber with a weak doping concentration (the absorption coefficient at $1535 \mathrm{~nm}$ was $<0.01 \mathrm{~cm}^{-1}$ ) and a pump power of $17 \mathrm{~mW}$ at 


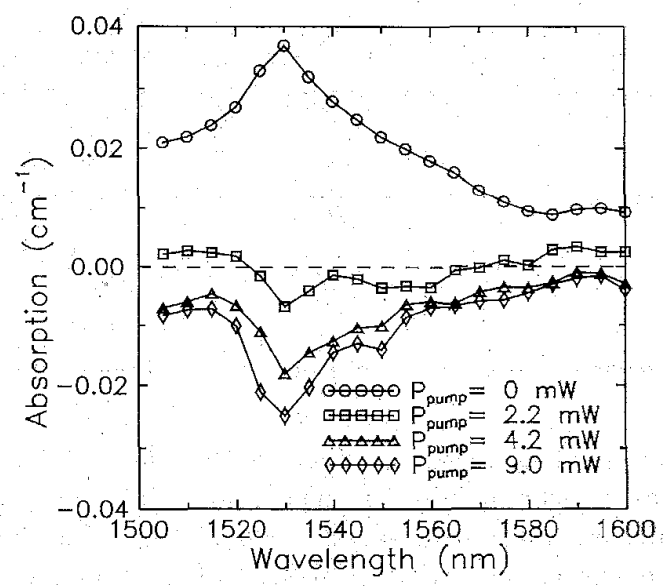

(a)

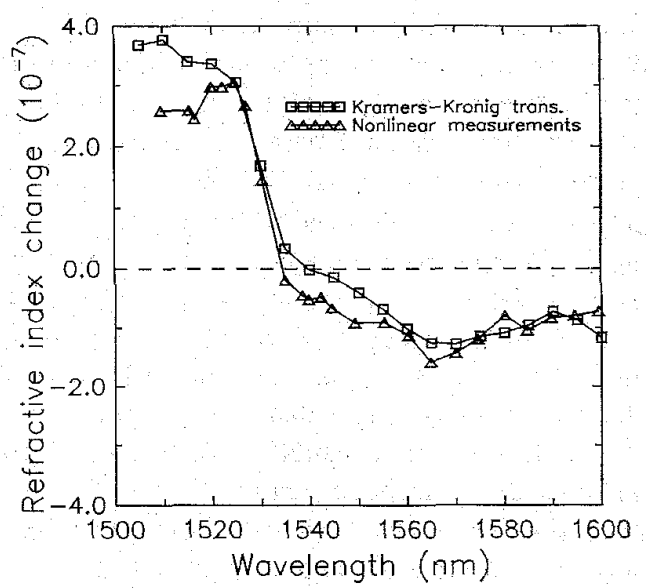

(b)

Fig. 4. (a) Absorption as function of wavelength for fiber $A\left(\mathrm{Er}^{3+}{ }_{\text {-doping }}\right.$ concentration of $\left.0.7 \times 10^{19} \mathrm{~cm}^{-3}\right)$ at an absorbed pump power of $0 \mathrm{~mW}$ (circular symbols), $2.2 \mathrm{~mW}$ (square symbols), $4.2 \mathrm{~mW}$ (triangular symbols) and $9.0 \mathrm{~mW}$ (diamond symbols); (b) Kramers-Kronig transform on the change in absorption for a change in absorbed pump power from 0 to $2.2 \mathrm{~mW}$ (square symbols) and direct measurement of the refractive index change (triangular symbols) as function of wavelength. An offset of $0.8 \times 10^{-7}$ has been added to the data obtained from the Kramers-Kronig transtorm.

$980 \mathrm{~nm}$. The authors interpreted the offset to be due to thermal effects. Pantell et al. [5] have measured the pump induced phase change indirectly from the switching characteristics of a twin core fiber with an $\mathrm{Er}^{3+}$ doping concentration of $1.3 \times 10^{19} \mathrm{~cm}^{-3}$ using a pump wavelength of $1480 \mathrm{~nm}$ and a signal wavelength of $980 \mathrm{~nm}$, and they measured an offset of $1.5 \mathrm{rad}$ which is about $80 \%$ of a measured peak to trough value of $1.8 \mathrm{rad}$. The authors interpreted the offset to be due to high energetic optical transitions for $\operatorname{Er}^{3+}$. For the cases shown in Figs. 3 and 5 with various pump powers and doping concentrations, the offsets are only 17 to $24 \%$ of the peak to trough pump induced refractive index change implying smaller offsets for the measurements reported here.

Fig. 6(a) shows the refractive index change $\Delta n$ as function of absorbed pump power for fiber $A$ and $C$ at a wavelength on the short wavelength side of resonance where $\Delta n$ is positive and at a wavelength on the long wavelength side where $\Delta n$

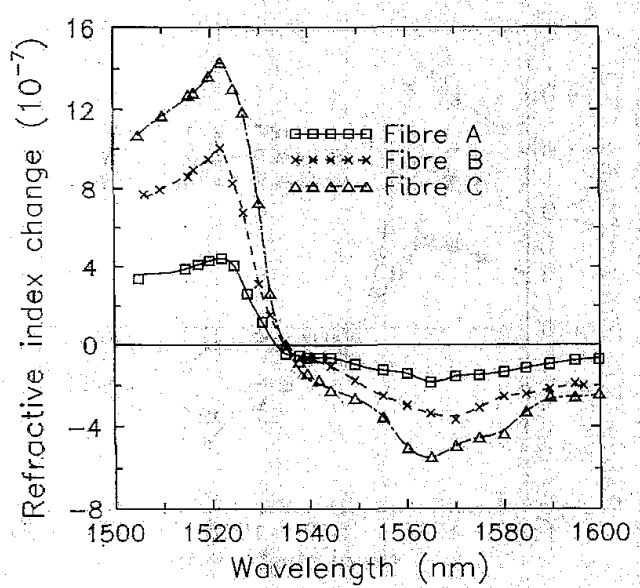

(a)

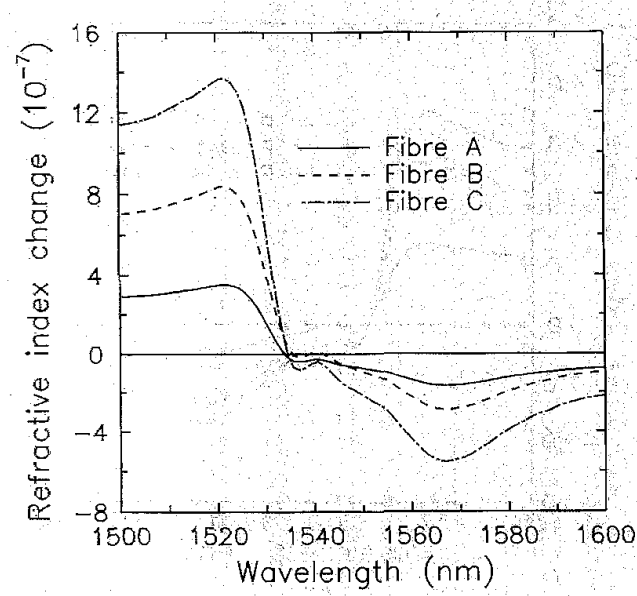

(b)

Fig. 5. Measured (a) and calculated (b) refractive index change as func tion of wavelength for fiber $A$ with an $\mathrm{Er}^{3}+$-doping concentration of $0.7 \times 10^{19} \mathrm{~cm}^{-3}$ (squate symbols and solid curves), fiber $B$ with an $\mathrm{Er}^{3+}$-doping concentration of $2 \times 10^{19} \mathrm{ctr}^{-3}$ (cross symbols and dashed curves) and fiber $C$ with an $\mathrm{Er}^{3+}$-doping concentration of $8 \times 10^{19} \mathrm{~cm}^{-3}$ (triangular symbols and dashdotted curves) The absorbed pump power at 980 $\mathrm{nm}$ is $4.2 \mathrm{~mW}$. The threshold pump power $P_{t h}$ bas been set equal to 08 $\mathrm{mW}$ for fiber $A$ and $B$ and equal to $1.5 \mathrm{~mW}$ for fiber $C$ The offsets for the calculated solid, dashed, and dashdotted curves in (a) have been set equal to $1.1 \times 10^{-7}$ for fiber $A, 3.0 \times 10^{-7}$ for fiber $B$ and $48 \times 10^{-7}$ for fiber $C$ (see text). These figures are 173,195 and $241 \%$ of the peak to trough induced $\triangle n$, respectively.

is negative. The solid and the dashdotted curves if Fig. 6(a) have being obtained from the model described in section IIIA where the offsets in $\Delta h$ for the two fibers have been calculated by fitting the calculated $\Delta n$ to the experimental data. In the calculations, the threshold pump powers $P_{\text {th }}$ have been set to the same values as in Fig. 5(b). The results of the calculated offsets are shown in Fig. 6(b). In Fig. 6(c), the corresponding ASE for the two fibers is plotted as function of absorbed pump power.

It is observed that even though (1) is expected to hold only in the unsaturated regine $[8], \mathrm{a}$ good fit to experimental data is obtained from the model for all the pump powers in Fig. $6(\mathrm{a})$. For fiber $A$ with low $\mathrm{Ex}^{3+}$-concentration, $\Delta n$ increases until an absorbed optical power of $\sim 5-10 \mathrm{~mW}$ where it saturates. 


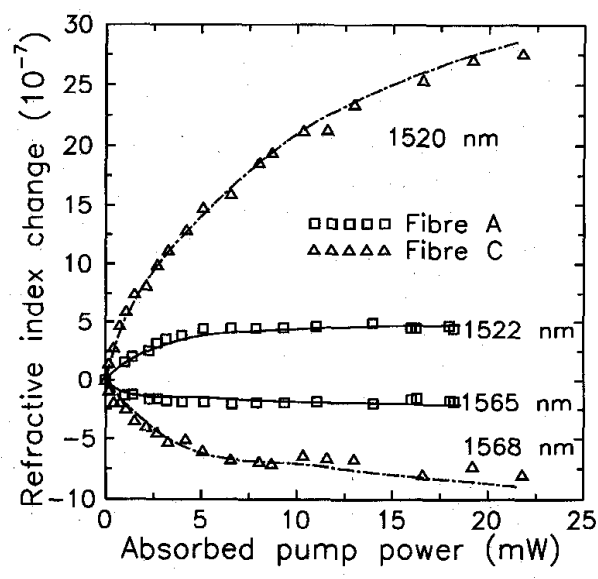

(a)

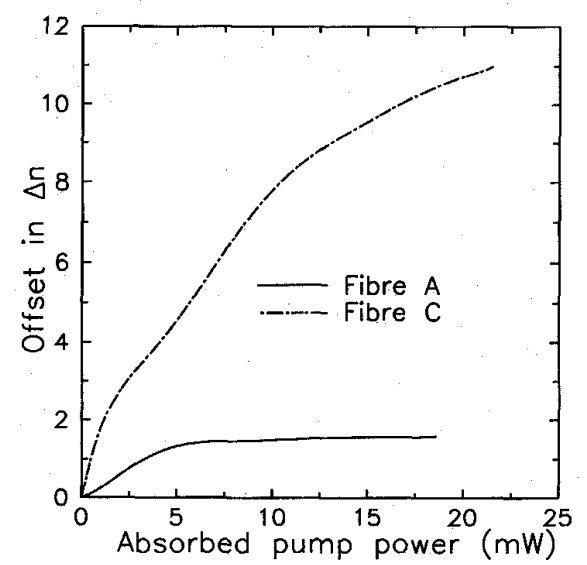

(b)

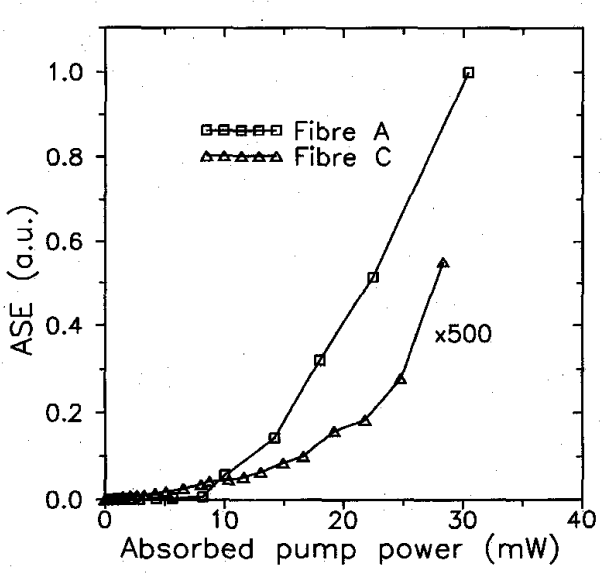

(c)

Fig. 6. (a) Refractive index change $\Delta n$ as function of absorbed pump power for fiber $A$ with an $\mathrm{Er}^{3+}$-doping concentrations of $0.7 \times 10^{19} \mathrm{~cm}^{-3}$ (square symbols) and fiber $C$ with an $\mathrm{Er}^{3+}$-concentration of $8 \times 10^{19} \mathrm{~cm}^{-3}$ (triangular symbols) at different wavelengths as indicated with the dashed and solid curves being obtained from the model described in the text; (b) the corresponding calculated offsets in $\Delta n$; and (c) the corresponding amplified spontaneous emission where the results for fiber $C$ [triangular symbols] have been multiplied by a factor of 500. The threshold pump power $P_{t h}$ for the two fibers have been set to the same values as in Fig. 5 .
For the highly doped fiber $C$ there is no saturation for absorbed pump powers up to at least $22 \mathrm{~mW}$. The offset in $\Delta n$ [see Fig. 6(b)] is observed to have a similar functional dependence on the pump power as $\Delta n$ in Fig. 6(a), suggesting that the offset originates from high energetic optical transit ons and is due to either changes in the absorption or nonradiative relaxation to the metastable energy level causing heating in the fiber.

In Fig. 6(c), it is observed that the ASE for the long but weakly doped fiber $A$ is low for an absorbed optical power below 5-10 $\mathrm{mW}$ and increases rapidly for higher optical powers. The ASE for the short but the highy doped fiber $C$ is more than 500 times less than for fiber $A$ for optical powers larger than $10 \mathrm{~mW}$. For fiber $A$, the 1 hreshold value for the ASE is similar to the saturation power for the optical nonlinearity [see Fig. 5(a)]. This can be interpreted as follows. A further increase in absorbed pump power will not cause increasing population of the metastable energy level responsible for the refractive index change, but the level will be emptied for carriers due to the ASE. For fiber $C$ [tiangular symbols in Fig. 5(a)], no threshold is observed for the ASE which is in agreement with the fact that no saturation in the change in the refractive index occurs for absorbed pump powers less than $22 \mathrm{~mW}$.

From the slopes of the curves in Fig. 6(a), a value of the cross phase optical nonlinearity $n_{s p}$ can be estimated. Using the values of $\Delta n$ for an optical power of $1 \mathrm{~mW}$ and sissuming that the area of the light beam is equal to the area of the fiber core $\left(\sim 4 \times 10^{-7} \mathrm{~cm}^{2}\right)$, the results are: $n_{s p}==0.45 \times$ $10^{-10} \mathrm{~cm}^{2} / \mathrm{W}$ for fiber $A$ and $n_{s p}=1.1 \times 10^{-10} \mathrm{~cm}^{2} / \mathrm{W}$ for fiber $C$, where the contributions from the offsets have been subtracted. These nonlinearities derived from Fig. 6(a) are slightly larger than the figures calculated from the two level laser model (see section IIIB).

In an optical communication system comprising $\mathrm{Er}^{3+}$. doped fiber amplifiers, the wavelength dependent refractive index modulation induced by the pump gives rise to dispersion that can be accumulated along the system and affect the signal pulse. The dispersion is defined as

$$
D=-\frac{\lambda}{C} \frac{d^{2} \Delta n}{d \lambda^{2}}
$$

where $\Delta n$ is the pumped induced change in the refractive index, $c$ is the velocity of light in vacuum, and $\lambda$ is the wavelength of the signal. The dispersion is plotted in Fig. 7 as function of wavelength for fiber $A$ (solid curve), fiber $B$ (dashed curve), and fiber $C$ (dashdotted curve). The absorbed pump power is $4.2 \mathrm{~mW}$ for each fiber. The curves are obtained by applying the above expression for $D$ to the corresponding solid, dashed, and dashdotted curves for the experimental data of $\Delta n$ in Fig. 5(a). It is observed that just below and above the resonant wavelength $\lambda_{0} \sim 1530 \mathrm{~nm}$, the disfersion is maximum and in the range $50-250 \mathrm{ps} / \mathrm{nm} / \mathrm{km}$, and it is largest for the fiber with the highest doping concentration. However, if the operating wavelength is $\geq 20 \mathrm{~nm}$ below or above resonance, the dispersion is decreased significantly. Thus, at an operating wavelength of $1550 \mathrm{~nm}$, the absolute value of the dispersion is less than $10 \mathrm{ps} / \mathrm{nm} / \mathrm{km}$ for all thr ze fibers. 


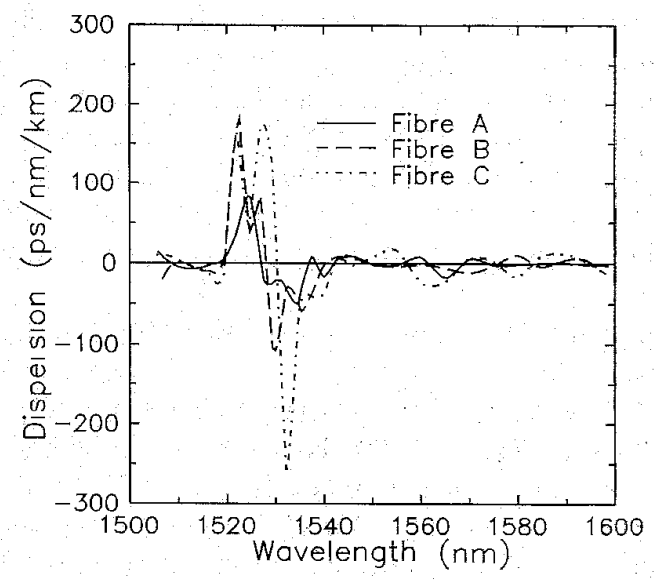

Fig. 7. Dispersion as function of wavelength due to pump induced change in the refractive index for fiber $A$ (solid curve), fiber $B$ (dashed curve) and fiber $C$ (dashdotted curve). The results are obtained from the corresponding solid, dashed and dashdotted curves in Fig. 5(a).

According to Fig. 4(a), the improved performance with regard to dispersion is obtained with penalty in amplification.

\section{CONCLUSIONS}

In this paper, an improved measurement system based on a Mach-Zehnder interferometer for determining pump induced phase changes in erbium doped fibers has been reported. The measurement technique yields an accuracy of $\sim \pi / 20$ and it is relatively insensitive to variations in external parameters such as power levels and polarization states in the two arms of the Mach-Zehnder interferometer. A systematical investigation of dependence of pump induced refractive index changes in erbium doped fibers upon wavelength, pump power, and $\mathrm{Er}^{3+}$-concentration was presented. It was observed that the fiber with the highest doping concentration yields the largest pump-induced refractive index changes and lowest undesirable amplified spontaneous emission. Fairly good agreement was obtained for refractive index changes measured directly by the method reported and values derived from Kramers-Kronig transform performed on spectra of pump-induced changes in the absorption. Apart from a wavelength independent offset in $\Delta n$, a model based on measured spectra of the absorption and emission cross sections yields good agreement to experimental results for variations in wavelength, pump power, and doping concentration. The offset was interpreted to originate from high energetic optical transitions and being due to either changes in the absorption or nonradiative relaxation to the metastable state causing heating in the fiber. Relatively to the peak to trough value of $\Delta n$, the offset was observed to increase with increasing doping concentration being $17 \%$ for the fiber with the lowest doping concentration $\left(0.7 \times 10^{19} \mathrm{~cm}^{-3}\right)$ and increasing to $24 \%$ for the fiber with the highest doping concentration $\left(8.0 \times 10^{19} \mathrm{~cm}^{-3}\right)$. Compared to other $\mathrm{Er}^{3+}$ doped fibers reported in the literature these offsets are small.

The results showed that a highly doped fiber yields the highest refractive index modulation with the limited effect of ASE. In applications where the dispersion is unwanted, a tradeoff between gain and refractive index modulation should be made for the operating wavelength. Close to resonance at $1530 \mathrm{~nm}$, the dispersion could be as large as $250 \mathrm{ps} / \mathrm{nm} / \mathrm{km}$, but below resonance at $1550 \mathrm{~nm}$, the dispersion was measured to be below $10 \mathrm{ps} / \mathrm{nm} / \mathrm{km}$ for the fibers investigated. The direct measurement technique with the high accuracy can be used in nonlinear measurements of some other doped fibers, such as semiconductor doped glasses and phosphorous oxygen hole center fibers, which may be important for optical switching applications.

\section{REFERENCES}

[1] S. C. Fleming and T. J. Whitley "Measurement of pump induced refractive index change in erbium doped fiber amplifier," Electron Lett., vol. 27, pp. $1959-1961,1991$

[2] R. W. Sadowski, M. J. F, Digonnet, RH Pantell, and H.J, Shaw, "Resonantly-enhanced nonlinear switching in rare earth doped fibers." in Proc SPIE, Boston, MA, Sept 1992.

[3] P. L. Chi and B. Wi, "Optical switching in twin-core erbium-doped fibers," Opt. Lett., vol. 17, pp. $235-257,1992$

[4] Y. L. Xue, P. L. Chu, and Weiping Zhang, "Resonance-enhanced refractive index and its dynamics in rare-earth-doped fibers, $J$ Opt $S o c$ $A m$. B, vol. 10, pp. $1840-1847,1993$.

[5] R. H. Pantell, M. J. F. Digonnet, R. W. Sadowski, and H. J. Shaw, "Analysis of nonlinear optical switching in an erbium-doped fiber," $J$ Lightwave Technol, vol. 11, pp. $1416-1424,1993$.

[6] M. J. F. Digonnet, R. W. Sadowski, D.T Mayweather, H. I Shaw, and R. H. Pantell, "Advances toward practical all-optical fiber switches," in Proc. Optic. Fiber Commun, 95 (OFC 95), San Diego, CA, Technical Digest, pp. 15-16.

[7] J. N. Sandoe, P. H. Sarkies, and S. Sparke, Variation of Er ${ }^{3}$ cross section for stimulated enission with glass composition," $J, P h y s . D$ vol. 5, pp. $1788-1799,1972$.

[8] E. Desurvire, "Study of the complex atomic susceptibility of erbium- doped fiber amplifiers," J. Lightwave Technol, vol 8, pp 1517-1527, 1090.

[9] F. Stern, "Dispersion of the index of refraction near the absorption edge of semiconductors," Phys. Rev, 133, pp A 1653 -A1664, 1964.

[10] A. Yariv, Optical Electronics, Saunders College Publishing, Fourth Ed Orlando, FL 1991

[11] G. I. Stegeman, E. M. Wright, N. Finlayson, R Zanoni, and C. T. Seaton "Third order nonlinear integrated optics," J. Lightwave Technol, yol. 6 , pp. 953-970, 1988.

[12] J. S. Weiner, D. A. B. Miller, and D. S. Chemla, Quadratic electrooptic effect due to the quantim-confined Stark effect in quantum wells? Appl. Phys. Lett., vol. 50, pp. 842 844, 1987

[13] R. Wyatt, "Spectroscopy of rare earth doped fibers" in Proc. SPIE, 1171, pp. 5464,1989 :

[14] B. I. Ainslie, S. P. Craig-Ryan, S. T Davey, I R Armitage, C.A Atkins, and R. Wyatt, "Optical analysis of erbium doped fibers for efficient lasers and amplifiers," in Proc. $1 O O C$, Kobe, Japan, 1989 $20 \mathrm{~A} 3-2$.

[15] N. Kagi, A. Oyobe, and K. Nakamura, Efficient optical amplifler using a low-concentration exbium-doped fiber" IEEE Photon. Technol Lett, vol. 2 ; pp. $559-561,1990$.

[16] H. Masuda, A. Takada, and K. Aida "Modeling the gain degradation of high concentration erbium doped fiber amplifiers by tntroducing inhomogerieous cooperative up-conversion," J. Lightwave Technol, vol 10, pp. $1789 \div 1799,1992$.

Carsten Thirstrup, photograph and biography not available at the time of publication.

Yuan Shi, photograph and biography not available at the time of publication

Bera Pálsdóttir, photograph and brography not available at the time of publication. 